\title{
Multiple axonal tracing: simultaneous detection of three tracers in the same section
}

Received: 9 June 1998

\begin{abstract}
Multiple neuroanatomical tract-tracing methods are important tools for elucidating the connectivity between different populations of neurons. Evaluation of the question as to whether two specific fiber inputs converge on a particular, identified population of projection neurons requires the application of a triple-staining procedure that allows the unequivocal detection of three markers in a single section. The present report deals with a combination of tracing methods using anterogradely transported Phaseolus vulgaris leucoagglutinin and biotinylated dextran amine in conjunction with retrogradely transported Fluoro-Gold. These tracers were simultaneously detected according to a three-color paradigm, which includes the use of three different peroxidase substrates (nickel-enhanced diaminobenzidine, diaminobenzidine, and Vector ${ }^{\circledR}$ VIP), thus resulting in three distinct precipitates: black, brown, and purple. We illustrate this method by showing convergence of projections arising from neurons located in two separate basal ganglia-related nuclei onto identified thalamostriatal projection neurons.
\end{abstract}

Key words Tract-tracing $\cdot$ PHA-L · BDA · Fluoro-Gold . V-VIP · Peroxidase.

\section{Introduction}

Axonal tracers are powerful tools for the study of neuronal circuits. Tract tracing allows us to study the way in which two or more brain regions are connected. New

J.L. Lanciego ( E. Erro · J.M. Giménez-Amaya Departamento de Anatomía, Facultad de Medicina, Universidad de Navarra, Irunlarrea s/n, E-31080 Pamplona, Spain e-mail: jlanciego@unav.es

Tel.: 34-48-425600 ext. 6235; Fax: 34-48-425649

\section{F.G. Wouterlood}

Graduate School Neurosciences Amsterdam,

Research Institute Neurosciences Vrije Universiteit,

Department of Anatomy and Embryology, Faculty of Medicine,

Vrije Universiteit, 7 van der Boechorststraat,

1081 BT Amsterdam, The Netherlands tools for tract tracing are continuously being developed. In the past few years major efforts have been directed toward the design of protocols combining more than one tracer.

Several technical problems may arise when designing protocols simultaneously combining a few tracers. Firstly, multiple protocols have to include tracers with a similar nature (fluorescent tracers versus non-fluorescent markers). Secondly, the need to deal with tracers with comparable survival times is mandatory in order to avoid difficult or repetitive surgical approaches. Axonal tracers such as horseradish peroxidase (HRP; Kristensson et al. 1971; Mesulam 1982) or biocytin (King et al. 1989; Izzo 1991; Lachica et al. 1991; Norita et al. 1991; Sørensen et al. 1993) only require 1 or 2 days of survival time, while other markers such as Phaseolus vulgaris leucoagglutinin (PHA-L) and biotinylated dextran amine (BDA) have a wider spectrum of survival time (Gerfen and Sawchenko 1984; Ter Horst et al. 1984; Brandt and Apkarian 1992; Veenman et al. 1992; Rajakumar et al. 1993; Reiner et al. 1993; Wouterlood and Jorritsma-Byham 1993; Lanciego and Wouterlood 1994) ranging from just a few days to 3 weeks in the case of BDA, or even few months when considering PHA-L (Groenewegen and Wouterlood 1990; Wouterlood and Groenewegen 1991). The range of survival time of fluorescent tracers (Wessendorf 1990) such as Fast Blue, Diamidino Yellow, Fluoro-Gold (FG; Schmued and Heimer 1990) and many others, are much more similar, but the fluorescent nature of these dyes hampers the combination of these with other tracers. In this respect, the introduction of an excellent antibody raised in rabbit against FG (Chang et al. 1990, Van Bockstaele et al. 1994) opens a whole range of possibilities for the application of this tracer in combination with other markers under standard light microscopy. Another source of limitations when combining axonal tracers lies in the possible appearance of crossreactivity phenomena between the different antisera employed to detect the different markers. Many commercial antibodies raised in a large number of animal species have already been introduced, which makes it easier to circumvent 
crossreactivity. Finally, the number of peroxidase substrates currently available is relatively small, and a few of them are difficult to handle and are often carcinogenic, or suspected to be carcinogenic, and thus quite unsafe to be used. The recent introduction of the new peroxidase substrate Vector ${ }^{\circledR}$ VIP (V-VIP; forms a purple precipitate), can be an excellent choice for circumventing this particular problem (Zhou and Grofova 1995; Lanciego et al. 1997a).

In order to elucidate the microcircuits involved in pathways interconnecting the basal ganglia, we needed to develop a triple-staining paradigm, combining two anterograde axonal tracers such as PHA-L and BDA, with retrograde axonal tracing using FG. This sensitive procedure would allow us to study the degree of convergence of two different sets of projections, arising from two separate basal ganglia-related nuclei, within the thalamus, and more specifically, to determine whether or not these inputs are in contact with the thalamostriatal neurons, labeled retrogradely using FG. As a result of the three-color paradigm described here, we are able to detect all of these three tracers (BDA, PHA-L, and FG) in a single section, using three different peroxidase substrates [nickel-enhanced diaminobenzidine (DAB-Ni), diaminobenzidine (DAB), and V-VIP. A preliminary report (Lanciego et al. 1997b) has been presented in abstract form.

\section{Materials and methods}

All experiments were carried out on female Wistar rats ( $n=9$; body weight 210-250 g), deeply anesthetized with an intramuscularly injected mixture of 4 parts of Ketaset (1\% of a solution of ketamine; Aesco, Boxtel, The Netherlands) to 3 parts of Rompun (2\% solution of xylazine, Bayer, Leverkusen, Germany). In order to minimize animal suffering, the animals were handled at all times according to the European Communities Council Directive 86/609/EEC. The rats were placed in a stereotaxic frame and all the tracers were iontophoretically injected in a single surgical session. BDA (Molecular Probes Europe, Leiden, The Netherlands) was injected into the substantia innominata (SI; $n=7$ ) or into the tegmental pedunculopontine nucleus (PPTg; $n=2$ ) as a $10 \%$ solution in $0.01 \mathrm{M}$ phosphate buffer, $\mathrm{pH} 7.25$, through a glass micropipette (inner tip diameter $25-40 \mu \mathrm{m}$ ) using a positive-pulsed direct current ( $7 \mathrm{~s}$ on/off for $10 \mathrm{~min}$ ). Next, a $2.5 \%$ solution of PHA-L (Vector Laboratories, Burlingame, Calif., USA) in 0.05 M TRISbuffered saline (TBS), $\mathrm{pH}$ 7.4, was delivered into the ipsilateral substantia nigra (SN), using the same iontophoretic parameters described above for the BDA injections. Finally, a $2 \%$ solution of FG (Fluorochrome, Englewood, Calif., USA) in $0.1 \mathrm{M}$ cacodylate buffer, $\mathrm{pH}$ 7.3, was injected into the ipsilateral nucleus accumbens (Acb, $n=4)$ or into the ipsilateral striatum $(\mathrm{CPu}, n=5)$, using the same procedure as for both BDA and PHA-L injections. Stereotaxic coordinates were taken from the atlas by Paxinos and Watson (1986).

After a survival time of 7 days, the animals were deeply reanesthetized with an overdose of $10 \%$ chloral hydrate in distilled water and perfused transcardially. The perfusates consisted of a Ringer saline rinsing solution (at body temperature), immediately followed by $1000 \mathrm{ml}$ of cold fixative containing $4 \%$ paraformaldehyde, $0.1 \%$ glutaraldehyde, and $0.2 \%$ saturated picric acid in $0.125 \mathrm{M}$ phosphate buffer, $\mathrm{pH}$ 7.4. After the perfusion, the skull was opened and the brain removed and stored in a cryoprotective solution (Rosene et al. 1986) containing 2\% dimethylsulfoxide and $20 \%$ glycerin in $0.125 \mathrm{M}$ phosphate buffer, $\mathrm{pH}$ 7.4. Coronal sections (40 $\mu \mathrm{m}$ thick) were obtained and collected in $0.125 \mathrm{M}$ phosphate buffer, $\mathrm{pH}$ 7.4.

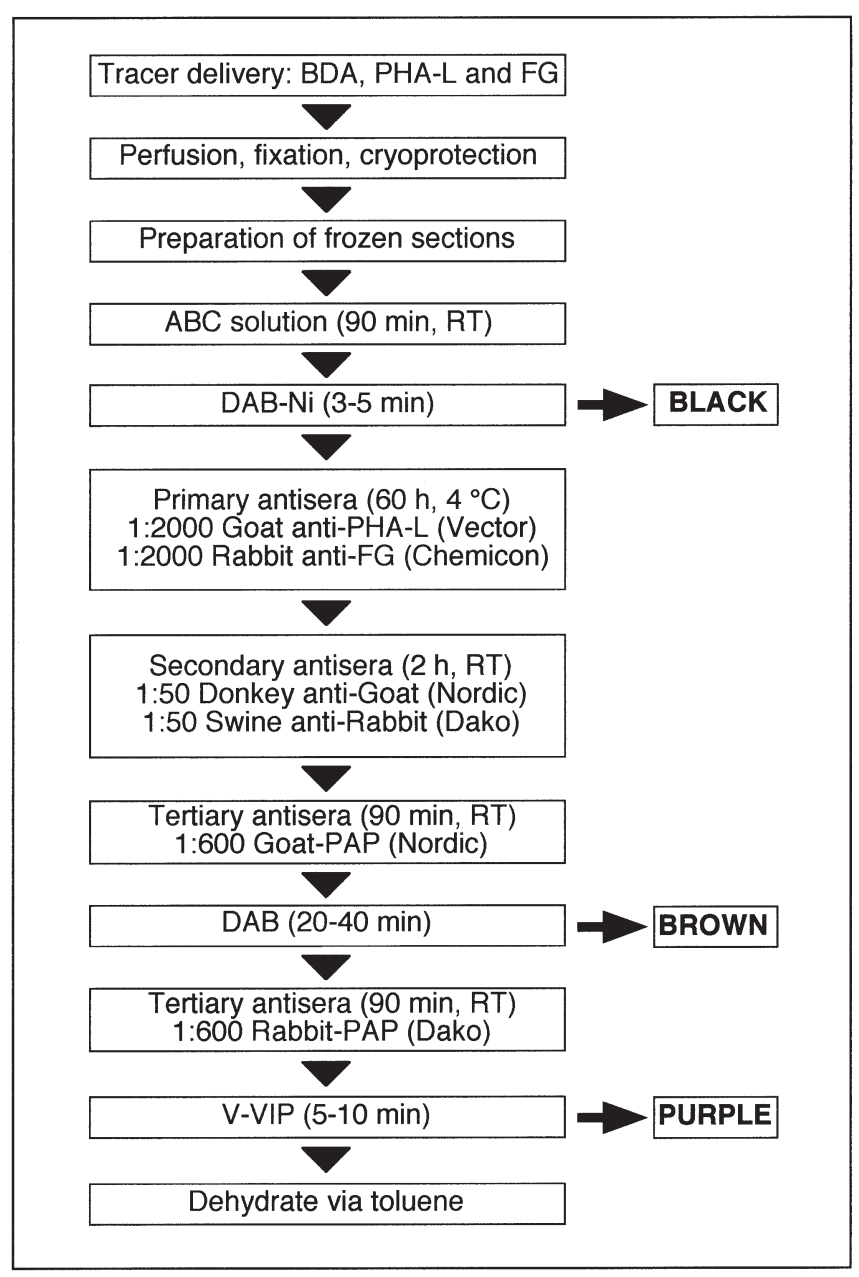

Fig. 1 Flow chart illustrating the various steps, antisera, and chromogens used in the present study. This sequential procedure allows us to detect in a single section: (1) anterogradely transported biotinylated dextran amine (BDA), stained black using nickel-enhanced diaminobenzidine (DAB-Ni) as a chromogen, (2) anterogradely transported Phaseolus vulgaris leucoagglutinin (PHA-L), stained brown with diaminobenzidine (DAB), and (3) retrogradely transported Fluoro-Gold (FG), stained purple using the novel peroxidase substrate Vector VIP (V-VIP). (PAP Peroxidase - anti-peroxidase, $R T$ room temperature)

The staining protocol described in the present paper deals with the simultaneous detection of three tracers in a single section according to a three-color paradigm, using three different peroxidase substrates. Obviously, these peroxidase substrates are not tracer specific, so one chromogen can be used for the detection of any of these three tracers. Several attempts were made testing the optimal chromogen for a particular marker. The procedure yielding the best results is described below (Fig. 1).

The BDA protocol (Veenman et al. 1992) was conducted using an $\mathrm{ABC}$ solution (ABC kit standard PK-4000; Vector) for $90 \mathrm{~min}$ at room temperature. Next, the sections were reacted for 5-10 min using DAB-Ni (Sigma, St. Louis, Mo., USA), resulting in a black precipitate. DAB-Ni incubation solution was prepared by dissolving $0.2 \mathrm{~g}$ of nickel ammonium sulfate and $7.5 \mathrm{mg}$ of DAB (Sigma) in $50 \mathrm{ml}$ of $0.05 \mathrm{M}$ TRIS/HCl, $\mathrm{pH} 8$. Immediately before use, $10 \mu \mathrm{l}$ of $30 \% \mathrm{H}_{2} \mathrm{O}_{2}$ was added. Subsequently, sections were incubated in a cocktail solution of primary antisera, comprising 1:2000 goat anti-PHA-L (Vector) and 1:2000 rabbit anti-FG [Chemicon, Temecula, Calif., USA; see Van Bockstaele et al. (1994) for more 
details regarding this antiserum], for $60 \mathrm{~h}$ at $4^{\circ} \mathrm{C}$. Incubation was continued in a solution containing 1:50 donkey anti-goat $\mathrm{IgG}$ (Nordic Immunological Laboratories, Tilburg, The Netherlands) and 1:50 swine anti-rabbit IgG (Dako, Copenhagen, Denmark) for $2 \mathrm{~h}$ at room temperature, and then incubated in a peroxidase-antiperoxidase (PAP) complex developed in goat (1:600 goat-PAP Nordic Immunological Laboratories) for $90 \mathrm{~min}$ at room temperature. The PHA-L protocol was then completed using DAB as a chromogen. DAB solution was prepared by dissolving $5 \mathrm{mg}$ of $\mathrm{DAB}$ in $10 \mathrm{ml}$ of $0.05 \mathrm{M}$ TRIS/HCl, pH 7.6, after which $3.3 \mu \mathrm{l}$ of $\mathrm{H}_{2} \mathrm{O}_{2}$ was added. The resulting solution was filtered prior to use Sections were incubated in this solution for 20-40 min and inspected in a microscope at regular time intervals. The final step of the FG protocol consisted of an incubation with a PAP complex raised in rabbit (1:600 rabbit-PAP, Dako) for $90 \mathrm{~min}$ at room temperature, followed by reaction for 5-10 min in V-VIP peroxidase substrate (Vector). The precipitate produced by this reaction has a purple color. V-VIP solution was prepared in $0.05 \mathrm{M}$ TRIS/HCl, $\mathrm{pH}$ 7.6, by using one drop of each vial (V-VIP is presented as a kit containing four vials) to prepare $3.5 \mathrm{ml}$ of working solution. Please note that no data exist as to the long-term effects from the use of V-VIP chromogen and as such it should be treated with the same care given the other chromogens. Once the staining was completed, the sections were mounted on glass slides using a $2 \%$ solution of gelatin in $0.05 \mathrm{M}$ TRIS/HCl $\mathrm{pH} 7.6$, dried at room temperature, quickly dehydrated in toluene, and coverslipped with Gurr ${ }^{\circledR}$ (BDH Laboratory Supplies, Poole, England). Throughout the different incubations in the respective chromogen solutions, sections were inspected in a microscope at 5 min intervals. All antisera used in this procedure were diluted in $0.05 \mathrm{M} \mathrm{TBS}, \mathrm{pH} 8$, with $0.5 \%$ Triton X-100 (Tx; Sigma). The incubations in the primary antisera were implemented with $2 \%$ of BSA (Merck, Darmstadt, Germany). Extensive washing with $0.05 \mathrm{M}$ TBS-Tx, $\mathrm{pH}$ 8, was carried out throughout the procedure. Several rinsing steps with $0.05 \mathrm{M}$ TRIS/HCl, $\mathrm{pH} 7.6$, were conducted prior to and after the reactions in the different chromogen solutions.

\section{Results}

Iontophoretic delivery of PHA-L (into the SN), BDA (into the SI or PPTg), and FG (into the Acb or $\mathrm{CPu}$ ) resulted in small, restricted injection sites (Fig. 2A-C). FG injection sites showed the largest deposits and in all cases it was possible to distinguish the effective uptake area from the surrounding halo. Fibers and terminals labeled anterogradely with PHA-L or BDA as well as FG-containing neurons were found in several thalamic nuclei (Fig. 2-H). The distribution of these projections was similar to that reported previously (Heimer et al. 1995; Gerfen and Wilson 1996).

The three-color paradigm used to detect the three tracers involved in this experimental paradigm showed an excellent quality of contrast. The colors of the related precipitates (black, brown, and purple) finally obtained had enough separation in the visible light spectrum to distinguish the identity of labeled fibers and cells unequivocally, independent of the magnification used (Fig. 2). According to our experimental design, the best resolution was obtained using first the DAB-Ni chromogen for the BDA stain, then the DAB substrate to complete the PHA-L protocol, and finally the V-VIP substrate for the detection of the FG-labeled neurons.

The degree of background stain can be considered to be very low for a triple-staining procedure (Fig. 2), as compared to the amount of background obtained in the single- or double-tracing protocols of earlier experiments. Two issues play key roles concerning this. Firstly, the consequent use of BSA as a component in the incubation of the primary antiserum clearly reduces the background staining, as we could appreciate by comparing the staining quality obtained in our earlier cases (incubated without BSA) with the background obtained in our last nine cases, all of them treated with $2 \%$ BSA during the incubation in the primary antisera. Secondly, the use of higher dilutions of the V-VIP substrate (as compared to the original instructions provided by the manufacturer), resulted in a more manageable substrate, because the way in which the reaction progresses was slower and thus easier to control under the microscope. Higher amounts of non-specific background stain were noticed when DAB-Ni was used to complete the PHA-L protocol, as compared to the background obtained when a regular solution of DAB was used to detect the PHA-L-labeled fibers.

\section{Discussion}

The recent introduction of a novel peroxidase substrate (V-VIP, forms a purple precipitate), as well as the availability of an antibody against FG raised in rabbit (Chang et al. 1990; Van Bockstaele et al. 1994), made it possible to simultaneously detect in a single section anterogradely transported BDA and PHA-L, and retrogradely transported FG according to a three-color paradigm. We applied this paradigm with success in our studies dealing with the convergence of inputs arising from two basal ganglia-related nuclei onto single, identified, thalamostriatal projection neurons.

\section{Trouble-shooting}

\section{Color mixing}

A phenomenon that should be taken into account is the so-called "color mixing". This emerges as a slight instability in the color of the reaction product corresponding to the chromogen used first, when proceeding to the incubation for the second chromogen. When color mixing occurs, there is a tendency to take the color associated with the second chromogen in zones where the first one has been deposited. This holds true especially in areas with high concentrations of the first reaction product (injection sites and periinjection-labeled neurons). Color mixing can also occur with the first two chromogens of a triple stain when a third one is added and is observed quite often when one of the antigens requires long incubations with the chromogens to be detected. The only way to avoid this kind of phenomenon is by means of inspection in a microscope at regular time intervals during the progress of the incubations in the different chromogens in order to halt the reaction, just in time, before col- 
or mixing begins to appear. Another, natural possibility for the color-mixing phenomenon is when two of three antigens (or all three of them) are colocalized within the same structure (for example, a neuronal soma). The present experimental protocol ruled out this kind of color mixing. Finally, color mixing can be induced by crossre- actions between the different antisera used in multiplestaining protocols, and this possibility merits special attention when dealing with combinations aimed at simultaneously detecting three different markers. This possibility seems to be very unlikely in our procedures, since the BDA protocol was carried out with an $\mathrm{ABC}$ kit,
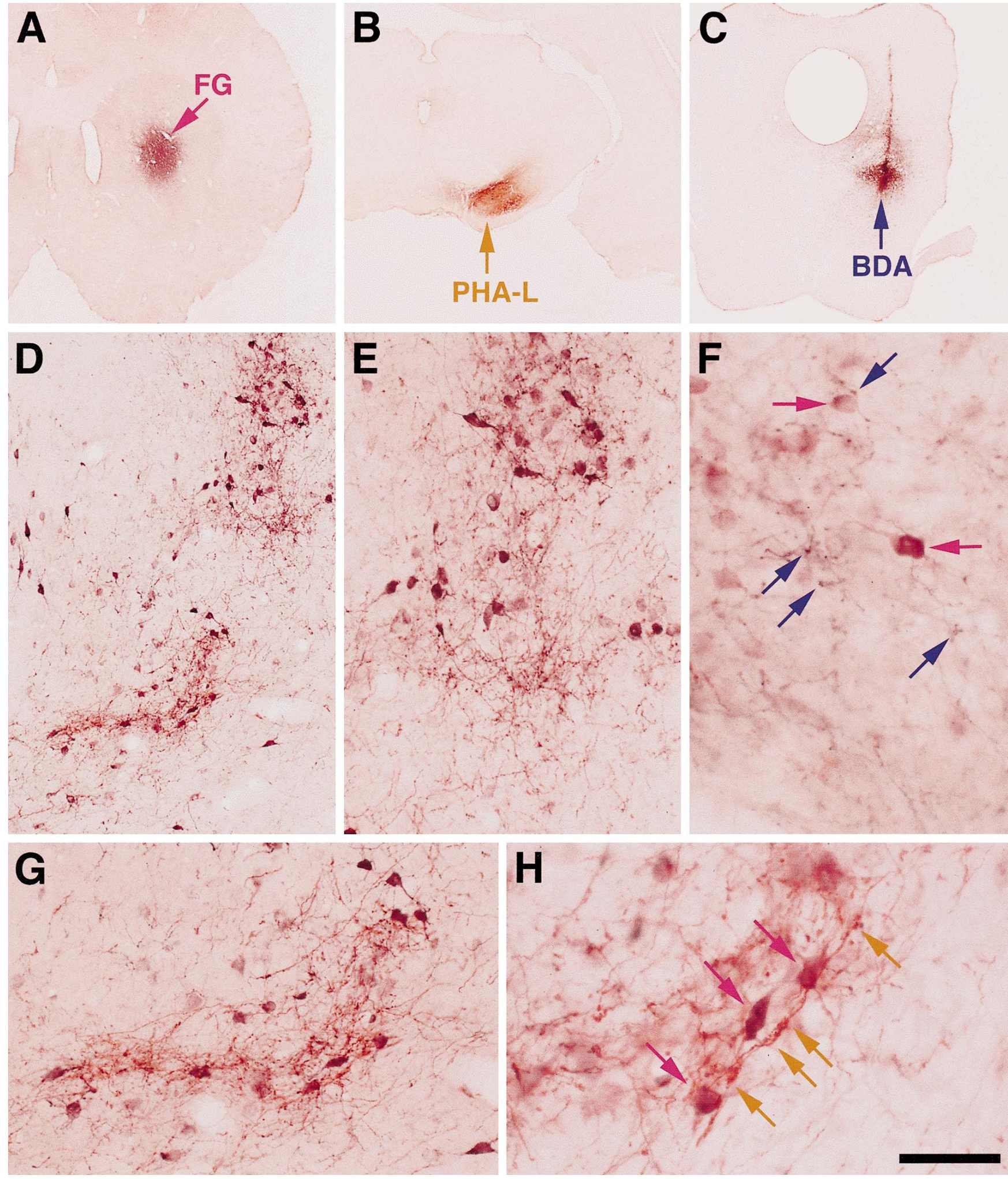

H 
whereas the antibody against FG was prepared in rabbit and the antibody against PHA-L was raised in goat. Separate controls showed that the rabbit and goat antisera did not cross-react.

\section{Background staining}

This phenomenon is of crucial importance when one aims at obtaining high technical quality in the results because it may hamper the contrast between the colors derived from the different reaction products of the chromogens used. The appearance of unacceptable degrees of non-specific background stain hinders the interpretation of the results of dual-staining protocols (Groenewegen and Wouterlood 1990), since it may prevent correct visualization of the finest structures labeled with a particular antigen. A low level of background stain is an important prerequisite in simultaneous triple-staining procedures. In our experience, the most important factor in avoiding unacceptable levels of background staining lies in stopping the incubations performed with the different chromogens in time. Careful microscopic control of the progress of these reactions is highly recommended because, if such care is not taken, the background stain obtained in the first incubation helps to enhance the background stain obtained in the second, and both will therefore have a multiplying effect on the background stain obtained in the incubation with the last chromogen. Additionally, the use of $2 \%$ BSA in the incubation media of the primary antisera resulted in lower degrees of background staining.

Fig. 2A-H Simultaneous detection of three tracers in a single section according to a three-color paradigm (black, brown, and purple). A Illustrates the FG injection site in the striatum, stained purple using the peroxidase substrate V-VIP. B PHA-L deposit in the substantia nigra. This anterograde tracer is detected via a PAP method and the reaction is completed with the peroxidase substrate DAB, resulting in a brown precipitate. $\mathbf{C}$ Photomicrograph showing an small, restricted $B D A$ injection site in the tegmenta pedunculopontine nucleus (PPTg). This anterograde tracer is stained blue-black using DAB-Ni as a chromogen. D-H Thalamus. D Photomicrograph showing the degree of convergence of inputs arising from PPTg (BDA-labeled fibers; black) and from the substantia nigra (PHA-L-labeled fibers; brown) onto identified thalamostriatal neurons (FG retrogradely labeled neurons; purple). This sequential protocol allows us to obtain an unequivocal distinction between the three tracers, even in brain areas in which the labeled structures overlap with a high density. Differences regarding the intensity of FG-labeled neurons (light purple versus dark purple) were probably due to different amounts of retrograde transport. E-H Areas of $\mathbf{D}$ at higher magnification in order to better illustrate the sensitivity of the present procedure. E, F An area of overlapping territories between the PPTg projections (BDA-labeled fibers; blue arrows) and somatopetally FG-labeled thalamostriatal neurons (purple arrows). G, H Convergence of nigrothalamic axons (PHA-L anterogradely labeled brown axons; brown arrows) onto identified thalamostriatal cells (FG-labeled; purple arrows). Bars A-C $2000 \mu \mathrm{m}, \mathbf{D} 200 \mu \mathrm{m}, \mathbf{E}, \mathbf{G} 100 \mu \mathrm{m}, \mathbf{F}, \mathbf{H}$ $50 \mu \mathrm{m}$
Selection of the most appropriate chromogen for a particular marker

According to the previous statements, the selection of the chromogen which is going to be used for the detection of a particular tracer is crucial. In the present experimental design we are dealing with a combination of two fiber stains (anterograde tracing with BDA and PHA-L) plus a stain for neuronal somata (retrograde tracing with FG). Since V-VIP induces a slight, intrinsic degree of non-specific background staining (Zhou and Grofova 1995; Lanciego et al. 1997a), we considered this substrate to be the most appropriate for cellular stains, while the lower background stain obtained with both DAB-Ni and DAB made these the most suitables chromogens for the anterograde fiber stains. Once V-VIP substrate had been chosen for the FG stain, the next decision was the assignment of either DAB-Ni or DAB for the detection of PHA-L- or BDA-labeled fibers, or vice versa. The use of DAB-Ni for the PHA-L stain always resulted in higher background staining, when compared to the background obtained in the PHA-L protocol when DAB was used as a chromogen. Another possible choice consisted of using DAB-Ni for the BDA stain, DAB to disclose PHA-L-labeled fibers, and V-VIP for the detection of the FG-labeled cells. This latter option allowed us to use both primary antisera (goat anti-PHA-L and rabbit antiFG) together in a cocktail solution as well as to use in sequence another cocktail solution including the secondary antisera (donkey anti-goat and swine anti-rabbit). Such a procedure significantly reduced the time spent in completing the entire triple-staining procedure (Lanciego et al. 1997a,b; 1998). There was almost no background stain after the use of DAB-Ni to complete the BDA protocol, probably due to the use of an $\mathrm{ABC}$ and the absence of any kind of antibodies involved in this histochemical procedure.

\section{Alternative protocols for triple staining}

The three-color paradigm described here has been successfully used for the simultaneous visualization of BDA and FG in combination with the immunocytochemical detection of the calcium-binding proteins parvalbumin or calbindin (Lanciego et al. 1997a). Moreover, this procedure can also be used for multiple neuroanatomical tracing in monkeys, combining anterograde tracing with BDA with dual retrograde tract tracing using cholera toxin B subunit and FG (Lanciego et al. 1998). Furthermore, V-VIP substrate is characterized by a specific, granular, electron-dense reaction product (Zhou and Grofova 1995). Although electron microscopy is not the primary goal of the present study, a method aimed at simultaneously combining BDA+PHA-L+FG (stained respectively with silver-enhanced DAB, regular DAB, and V-VIP) seems to be very attractive for further testing.

Very few protocols aimed at simultaneously detecting three tracers have been described in the literature. Smith 
and Bolam (1991, 1992) have reported a protocol combining retrogradely transported WGA-HRP with anterogradely transported PHA-L and biocytin. These tracers were respectively stained with TMB (blue), DAB-Ni (blue-black), and DAB (brown). This procedure was initially designed for electron microscopy, using $\mathrm{DAB}$ for the biocytin stain and BHDC for the PHA-L detection. At the light microscopic level, the above-mentioned sequence of chromogens does not afford a clear distinction of the labeled elements by color. Dolleman-Van der Weel et al. (1995) introduced a light microscopic sequential protocol for multiple axonal tracing, combining three anterograde axonal tracers including PHA-L, RDA, and $\mathrm{BDA}$, stained respectively with DAB-Ni (black reaction product), DAB (brown precipitate), and 1-naphthol/azur B (blue-green) as chromogens. 1-Naphthol/azur B is a chromogen initially reported as an alternative to DAB (Mauro et al. 1985). This protocol has two minor drawbacks. Firstly, it may be difficult to establish an unequivocal distinction between the three different tracers in areas where the three types of fibers concur massively, each one of them stained with a different chromogen. Secondly, fading of 1-naphthol/azur B reaction product occurs at room temperature, although the stain can be restored by a repeat incubation with the basic dye (Mauro et al. 1985).

The protocol presented here does not exhibit the aforementioned problems, thus making it always possible to clearly distinguish the differently labeled structures. Additionally, the present triple staining does not impose demands on the storage of the sections after staining. Only some specific caution has to be exerted. Firstly, one has to choose the most appropriate chromogen for the detection of a particular marker. Secondly, careful control under the microscope of the progress of the reactions is crucial to avoid color mixing as well as unacceptable degrees of background staining. Thirdly, toluene should be used for dehydration procedures, because the V-VIP substrate is highly unstable in standard treatment with ethanol (Zhou and Grofova 1995).

Acknowledgments Expert technical assistance was provided by Ms A. Moreno. Supported by F.I.S. No 96/0488, Fundación "Marcelino Botín" and University of Navarra (P.I.U.N.A.).

\section{References}

Brandt HM, Apkarian AV (1992) Biotin-dextran: a sensitive anterograde tracer for neuroanatomic studies in rat and monkey. J Neurosci Methods 45:35-40

Chang HT, Kuo H, Whittaker JA, Cooper NGF (1990) Light and electron microscopic analysis of projection neurons retrogradely labeled with Fluoro-Gold: notes on the application of antibodies to Fluoro-Gold. J Neurosci Methods 35:31-37

Dolleman-Van der Weel MJ, Wouterlood FG, Witter MP (1995) Multiple anterograde tracing, combining Phaseolus vulgaris leucoagglutinin with rhodamine- and biotin-conjugated dextran amine. J Neurosci Methods 51:9-21

Gerfen CR, Sawchenko PE (1984) An anterograde neuroanatomical tracing method that shows the detailed morphology of neurons, their axons and terminals: immunohistochemical local- ization of an axonally transported plant lectin, Phaseolus vulgaris leucoagglutinin (PHA-L). Brain Res 209:219-238

Gerfen CR, Wilson CJ (1996) The basal ganglia. In: Swanson LW, Björklund A, Hökfelt T (eds) Integrated systems of the CNS, part III. Handbook of chemical neuroanatomy, vol 12. Elsevier Science Publishers, Amsterdam, pp 369-466

Groenewegen HJ, Wouterlood FG (1990) Light and electron microscopic tracing of neuronal connections with Phaseolus vulgaris-leucoagglutinin (PHA-L) and combination with other neuroanatomical techniques. In: Wouterlood FG, Van den Pol A, Björklund A, Hökfelt T (eds) Handbook of chemical neuroanatomy, vol 8. Elsevier Science Publishers, Amsterdam, pp $47-124$

Heimer L, Zahm DS, Alheid GF (1995) Basal ganglia. In: Paxinos G (ed) The rat nervous system, 2nd edn. Academic Press, Sydney, pp 579-614

Izzo PN (1991) A note on the use of biocytin in anterograde tracing studies in the central nervous system: application at both light and electron microscopic level. J Neurosci Methods 36:155-166

King MA, Louis PM, Hunter BE, Walker DW (1989) Biocytin: a versatile anterograde neuroanatomical tract-tracing alternative. Brain Res 497:361-367

Kristensson K, Olson Y, Sjostrand J (1971) Axonal uptake and retrograde transport of exogenous proteins in the hypoglossal nerve. Brain Res 32:399-406

Lachica EA, Mavity-Hudson JA, Casagrande VA (1991) Morphological details of primate axons and dendrites revealed by extracellular injection of biocytin: an economic and reliable alternative to PHA-L. Brain Res 564:1-11

Lanciego JL, Wouterlood FG (1994) Dual anterograde axonal tracing with Phaseolus vulgaris leucoagglutinin (PHA-L) and biotinylated dextran amine (BDA). Neurosci Prot 94-050-06

Lanciego JL, Goede PH, Witter MP, Wouterlood FG (1997a) Use of peroxidase substrate Vector VIP for multiple staining in light microscopy. J Neurosci Methods 74:1-7

Lanciego JL, Wouterlood FG, Giménez-Amaya JM (1997b) Multiple axonal tracing: simultaneous detection of three tracers in one and the same section. 27th Annual Meeting of the Society for Neuroscience

Lanciego JL, Luquin MR, Guillén J, Giménez-Amaya JM (1998) Multiple neuroanatomical tracing in primates. Brain Res Prot 2(4):323-332

Mauro A, Germano I, Giaccone G, Giordana MT, Schiffer D (1985) 1-Naphthol basic dye (1-NBD), an alternative to diaminobenzidine (DAB) in immunoperoxidase techniques. Histochemistry 83:97-102

Mesulam MM (1982) Tracing neural connections with horseradish peroxidase. In: Mesulam MM (ed). Methods in the neurosciences. IBRO Handbook Series. Wiley and Sons. Chichester

Norita M, McHaffie JG, Shimizu H, Stein BE (1991) The corticostriatal and corticotectal projections of the feline lateral suprasylvian cortex demonstrated with anterograde biocytin and retrograde fluorescent techniques. Neurosci Res 10:149-155

Paxinos G, Watson C (1986) The rat brain in stereotaxic coordinates, 2nd edn. Academic Press, Sydney

Rajakumar N, Elisevich K, Flumerfelt BA (1993) Biotinylated dextran: a versatile anterograde and retrograde neuronal tracer. Brain Res 607:47-53

Reiner A, Veenman CL, Honig MG (1993) Anterograde tracing using biotinylated dextran amine. Neurosci Prot 93-050-14

Rosene DL, Roy NJ, Davis BJ (1986) A cryoprotection method that facilitates cutting frozen sections of whole monkey brains for histological and histochemical processing without freezing artifact. J Histochem Cytochem 34:1301-1316

Schmued LC, Heimer L (1990) Iontophoretic injection of fluorogold and other fluorescent tracers. J Histochem Cytochem 38:721-723

Smith Y, Bolam JP (1991) Convergence of synaptic inputs from the striatum and the globus pallidus onto identified nigrocollicular cells in the rat: a double anterograde labeling study. Neuroscience 44:45-73 
Smith Y, Bolam JP (1992) Combined approaches to experimental neuroanatomy: combined tracing and immunocytochemical techniques for the study of neuronal microcircuits. In: Bolam JP (ed) Experimental neuroanatomy, a practical approach. Oxford University Press, Oxford, pp 239-266

Sørensen JC, Tønder N, Zimmer J (1993) Biocytin pellets: an alternative technique for massive anterograde labeling of neuronal pathways in vivo and in vitro. Brain Res 608:338344

Ter Horst GJ, Groenewegen HJ, Karst H, Luiten PGM (1984) Phaseolus vulgaris leucoagglutinin immunohistochemistry. A comparison between autoradiographic and lectin tracing of neuronal efferents. Brain Res 307:379-383

Van Bockstaele EJ, Wright AM, Cestari DM, Pickel VM (1994) Immunolabeling of retrogradely transported fluorogold: sensitivity and application to ultrastructural analysis of transmitterspecific mesolimbic circuitry. J Neurosci Methods 55:6578
Veenman CL, Reiner A, Honig MG (1992) Biotinylated dextran amine as an anterograde tracer for single- and double-label studies. J Neurosci Methods 41:239-254

Wessendorf MW (1990) Characterization and use of multi-color fluorescence microscopic techniques. In: Björklund A, Hökfelt T, Wouterlood FG, Van den Pol AN (eds) Handbook of chemical neuroanatomy, vol 8. Elsevier Science Publishers, Amsterdam, pp 1-41

Wouterlood FG, Groenewegen HJ (1991) The Phaseolus vulgarisleucoagglutinin tracing technique for the study of neuronal connections. Prog Histochem Cytochem 22:

Wouterlood FG, Jorritsma-Byham B (1993) The anterograde neuroanatomical tracer biotinylated dextran amine: comparison with the tracer PHA-L in preparations for electron microscopy. J Neurosci Methods 48:75-87

Zhou M, Grofova I (1995) The use of peroxidase substrate Vector VIP in electron microscopic single and double antigen localization. J Neurosci Methods 62:149-158 